Case Report Journal of Epilepsy Research pISSN 2233-6249 / elSSN 2233-6257

Received November 14, 2020

Revised December 24, 2020

Accepted December 31, 2020

Corresponding author:

Hussein Algahtani, MD, FRCPC, MMed

Department of Neurology, King Abdulaziz

Medical City, King Saud bin Abdulaziz

University for Health Sciences, P.O. Box:

12723, Jeddah 21483, Saudi Arabia

Tel. +966-556633130

E-mail; halgahtani@hotmail.com

\section{Irreversible Cerebellar Atrophy as a Complication of Short-Term Phenytoin Exposure: Clinical Improvement Following Discontinuation of the Culprit}

\author{
Hussein Algahtani, MD, FRCPC, MMed ${ }^{1,2,3}$, Bader Shirah, MD²,3, \\ Abdulrahman J. Alqahtani, MD ${ }^{4}$, Adnan Q. Al-Malki, MD ${ }^{4}$ \\ ${ }^{1}$ Department of Neurology, King Abdulaziz Medical City, Jeddah; ${ }^{2}$ College of Medicine, King Saud bin Abdulaziz \\ University for Health Sciences, Jeddah; ${ }^{3}$ Research Office, King Abdullah International Medical Research Center, \\ Jeddah; ${ }^{4}$ College of Medicine, King Khalid University, Abha, Saudi Arabia
}

\begin{abstract}
Phenytoin (diphenylhydantoin) is a widely used antiepileptic drug for controlling both generalized and partial seizures. Reversible cerebellar symptoms, including cerebellar ataxia, have been recognized as an adverse event of phenytoin use for many years. On the other hand, cerebellar degeneration has been reported with chronic use in an epileptic patient treated with this drug. We are reporting an interesting case of phenytoin induced acute pan-cerebellar syndrome with cerebellar atrophy on neuro-imaging that improved many years after discontinuation of the drug. Discontinuation of phenytoin may give a chance for the patient to recover slowly, months after stopping the drug. It is very important for the attending neurologist to educate the patients and their families on some common clinical manifestations suggestive of drug toxicity and perform a regular follow-up and clinical examination at regular intervals.
\end{abstract} (2020;10:96-99)

Key words: Phenytoin, Cerebellum, Atrophy, Neuronal plasticity, Saudi Arabia

\section{Introduction}

Phenytoin (diphenylhydantoin) is a widely used antiepileptic drug that was introduced by Merritt and Putnam in 1983. ${ }^{1}$ It is used for controlling both generalized and partial seizures due to its stabilizing influence on neuronal membranes without causing depression of the central nervous system. It acts by prolonging the inactivated state of voltage-gated $\mathrm{Na}^{+}$channels and controlling the refractory period of neurons which results in the limitation of the repetitive firing of action potentials. In addition, high doses of phenytoin can reduce the calcium influx, inhibit glutamate, and facilitate gamma-aminobutyric acid (GABA). ${ }^{2}$ Reversible cerebellar symptoms, including cerebellar ataxia, have been recognized as an adverse event for phenytoin use for many years. ${ }^{3}$ In 1941, Aring and Rosenbaum ${ }^{4}$ have considered cerebellar ataxia as a landmark indicating the uppermost threshold of tolerance of patients toward the drug. On the other hand, cerebellar degeneration has been reported with chronic use in an epileptic patient treated with this drug. ${ }^{3}$ In 1958, Utterback ${ }^{5}$ has reported and contributed the first histological finding of irreversible cerebellar damage after phenytoin overdose. Reversible cerebellar symptoms in phenytoin use are not rare. Irreversible cerebellar damage and atrophy developed after short-term exposure to phenytoin may be infrequent. In this article, we are reporting an interesting case of phenytoin induced acute pan-cerebellar syndrome with cerebellar atrophy on neuro-imaging that improved many years after discontinuation of the drug. We postulate the mechanism behind this complication and its recovery and review the topic.

\section{Case Report}

A 45-year-old male, the fourth of seven living siblings, was apparently normal until the age of 18 years when he reported right side facial twitching followed by a generalized tonic-clonic seizure. During these episodes, he bit his tongue, rolled his eyes upward, and produced a frothy secretion from the angle of the mouth. Post-ictally, the patient was tired, sleepy, and confused for minutes to a couple of hours. He presented initially to a local secondary care hospital where magnetic resonance imaging (MRI) and electroencephalography 
(EEG) services were not available. He was apparently normal at birth following an uneventful gestation, and his neonatal history and subsequent development including school performance were also normal. His family history was unremarkable with no history of spinocerebellar ataxia, epilepsy, or genetic disorders. He does not drink alcohol, use illicit drugs, or take any medications (both over the counter and prescription). Initial computed tomography (CT) scan of the brain performed in the emergency department was normal. He was started on a phenytoin loading dose followed by an oral intake of $100 \mathrm{mg}$ three times daily. Unfortunately, one month following the onset of phenytoin therapy, the patient developed dysarthria, severe ataxia, imbalance, action tremor, and difficulty walking. On neurological examination, the patient was unable to stand or walk with severe ataxia involving both the trunk and all extremities. He also had nystagmus, dysarthria, and symmetrical postural and action tremor. The plasma phenytoin level was $360 \mu \mathrm{mol} / \mathrm{L}(90 \mu \mathrm{g} / \mathrm{mL}$ ) (recommend therapeutic range, $40-80 \mu \mathrm{mol} / \mathrm{L}$ [10 to $20 \mu \mathrm{g} / \mathrm{mL}$ ]). The dose of phenytoin was rapidly reduced to $100 \mathrm{mg}$ daily and subsequently stopped, and treatment with valproic acid $750 \mathrm{mg}$ twice daily was commenced. A serial assessment of phenytoin concentrations was done, and phenytoin concentration bounced back to nadir after 9 days. Later on, levetiracetam was gradually introduced up to 1,000 $\mathrm{mg}$ twice daily. The patient has developed no more seizures since that time. He was re-evaluated by a tertiary care center where several CT scans and MRI of the brain showed diffuse pan-cerebellar symmetrical atrophy with no structural congenital, traumatic, or ischemic changes. Routine EEG was repeated twice, which showed no evidence of epileptiform discharges. The patient refused admission for EEG telemetry monitoring. There were no metabolic abnormalities including liver dysfunction that could explain the highly toxic plasma level. Measurement of different vitamin levels including vitamin E, folate, and vitamin B12 was normal. Genetic assessment of different cerebellar genetic disorders including spinocerebellar ataxia using whole-exome sequencing was unremarkable. On discharge from the hospital 2 weeks later, he still had severe pan-cerebellar symptoms and signs with ataxic gait and inability to walk without support. He was bedbound for a period of 4 months and started on a physiotherapy program. Since then, there has been slow but continuous

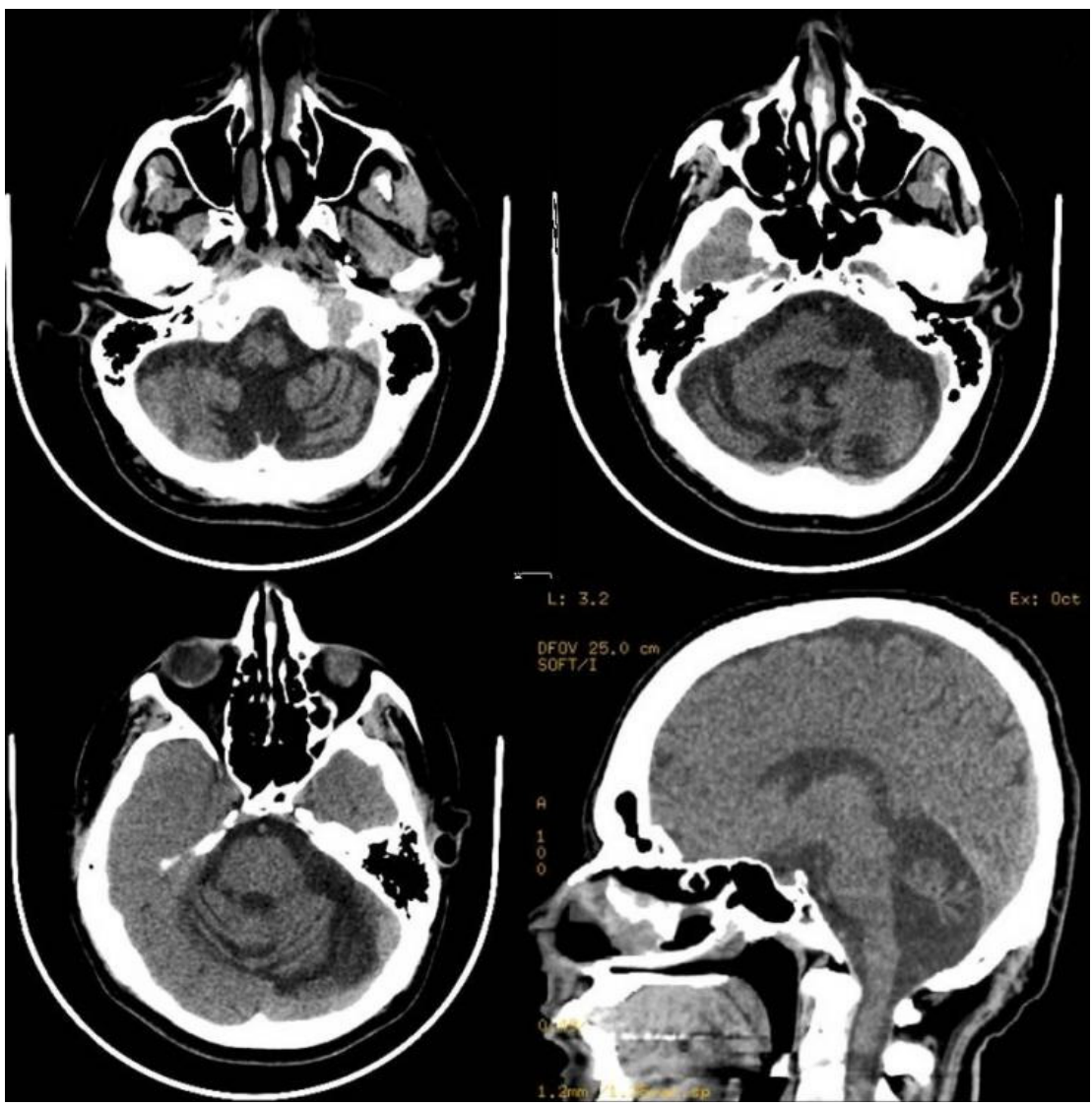

Figure 1. Computed tomography scan of the brain showing marked cerebellar atrophy and enlargement of the fourth ventricle and basal cisterns. 
gradual recovery with an improvement of all different cerebellar modalities including gait. Ten years after the intoxication, his condition improved remarkably, and the only abnormality was mild dysarthria and unsteadiness when running or turning rapidly. Despite the clinical improvement, a follow-up CT scan of the brain showed marked cerebellar atrophy and enlargement of the fourth ventricle and basal cisterns, which were similar to those images performed few months following initial presentation (Fig. 1).

\section{Discussion}

Phenytoin is an antiepileptic drug that was widely used for around 8 decades. It is a hydantoin aromatic antiepileptic medication with acceptable sedative properties. It is primarily used for the treatment of generalized tonic-clonic and partial seizures. The antiepileptic effect of this medication is achieved by the blockage of voltage-dependent neuronal $\mathrm{Na}^{+}$channels. This will increase the membrane threshold for depolarization and ultimately lower the neuronal cell susceptibility to epileptogenic stimuli. Specifically, it has a high selective inhibitory effect on the motor area of the cerebral cortex. In addition, it increases the level of certain neurotransmitters in the brain, mainly serotonin (5-HT) and GABA. The pharmacokinetic features of phenytoin are affected by three factors; the level of protein binding, liver metabolism, and non-linear drug elimination. Phenytoin is well absorbed in the duodenum following oral intake. It easily crosses the blood-brain barrier and the placenta, is extensively bound to plasma albumin (90\%), and is minimally excreted in breast milk. Phenytoin is metabolized by cytochrome P450 isoforms CYP2C9I/10 and CYP2C19. Due to competition from new antiepileptic drugs, complex kinetic profile, drug interactions, and long-term complications, the use of this medication is declining. ${ }^{6}$

Many dose-based adverse effects have been reported in the literature including sedation, visual disturbances, psychosis, muscle spasm, and cerebellar and vestibular toxicity. Adverse drug reactions at therapeutic doses include gum hypertrophy, hirsutism, and a large number of non-specific symptoms including fatigue, irritability, several involuntary functions, and loss of bodily movement. ${ }^{7}$ Cerebellar dysfunction due to phenytoin medication can be classified into acute and chronic. Acute phenytoin toxicity may cause ataxia, nystagmus, and other cerebellar signs which usually disappear when the dose is lowered or the drug is discontinued. These cerebellar symptoms and signs are usually transient with no structural damage seen on neuro-imaging. On the other hand, a chronic overdose has been found to cause cerebellar atrophy in adult patients using phenytoin. ${ }^{8}$
Phenytoin may cause cerebellar degeneration with the destruction of the Purkinje cells and a decline in the population of granule cells with Bergmann gliosis and relative sparing of basket cell axon of the cerebellum. Ultrastructural studies demonstrated a lamellar concentric body in the dendrites of Purkinje cells and the bodies of some basal cells. Neuro-imaging studies including MRI have also demonstrated diffuse cerebellar atrophy with special involvement of the cerebellar cortex. There is no correlation between the degree of atrophy, the severity of clinical symptoms, and the drug serum levels. ${ }^{9}$ In a study done by Shanmugarajah et al., ${ }^{10}$ cerebellar ataxia was present in $40 \%$ of patients with epilepsy and chronic exposure to phenytoin. In that study, 47 patients were recruited. Reduced cerebellar volume and evidence of structural deficit on imaging were observed. The pathomechanism of toxicity is thought by some researchers to be related to folate deficiency since phenytoin inhibits folate absorption from jejunal mucosa. This will impair hepatic drug-metabolizing capacity and neural functions.

The exact mechanism behind the improvement of cerebellar functions in our patient is not fully understood. It could be related to compensation or functional recovery following acute cerebellar damage. Although there is no histological, molecular, or other supportive evidence, neuroplasticity is another possible mechanism. This results in the sprouting of Purkinje cells' axon collaterals with the formation of heterotypical synapses with other Purkinje cells' dendritic spines. This may result in circuit re-organization with the replacement of the missing granule cells and glia and restitution of the normal circuitry. The release of endogenous chemical legends may also play a role. These findings were observed from studies on neuroplasticity in cerebellar culture models derived from newborn mice. ${ }^{11,12}$ Our patient improved clinically despite a lack of structural improvement in neuroimaging.

Although drug-induced ataxia is a relatively frequent occurrence in several antiepileptic drugs, cerebellar atrophy is not. Fosphenytoin is a phenytoin pro-drug that is rapidly metabolized and completely converted to phenytoin when administrated by intravenous or intramuscular injection. It is expected to have similar complications on the central nervous system including cerebellar atrophy. ${ }^{13}$ When reviewing the literature, we found only one case report of fosphenytoin-induced acute cerebellar dysfunction in a patient with acute myeloid leukemia. ${ }^{14}$ This could be related to the underutilization of fosphenytoin when compared to phenytoin. Pharmaco-economic consideration could be the main reason since fosphenytoin is 16 times more expensive than phenytoin. Another antiepileptic drug known to cause cerebellar atrophy is valproic acid. The first case of cerebellar 
atrophy was described in 1987 by McLachlan. ${ }^{15}$ Cerebellar shrinkage was reversible in their case.

Risk factors for the development of cerebellar atrophy in patients who receive phenytoin include higher doses, patients with CYP2 mutation, previous cerebellar injury, and patients with myoclonic type epilepsy. In addition, drug-drug interaction is another important risk factor since certain medications may increase the half-life of phenytoin and increase the chance of toxicity. ${ }^{16}$ Toxicity related to phenytoin may be hard to diagnose since the course could be chronic and gradual. ${ }^{17}$ A progressive cerebellar syndrome was described by Logan and Freeman ${ }^{18}$ in 1969. Although these cases may represent a field of diagnostic ambiguity, clinical examination including evaluation of coordination and gait should be practiced routinely with each patient visit to the clinic. In addition, exclusion of different genetic and acquired causes should be performed including genetic testing for spinocerebellar ataxia, different vitamin levels including vitamin $\mathrm{E}$, folate, and vitamin B12, and measurement of alcohol level in those who give a history of drinking alcohol.

Cerebellar atrophy may be seen in acute phenytoin toxicity in patients with epilepsy. Discontinuation of phenytoin may give a chance for the patient to recover slowly, months after stopping the drug. It is very important for the attending neurologist to educate the patients and their families on some common clinical manifestations suggestive of drug toxicity and perform a regular follow-up and clinical examination at regular intervals.

\section{Conflict of Interest}

The authors declare that they have no conflicts of interest.

\section{References}

1. Friedlander WJ. Putnam, Merritt, and the discovery of dilantin. Epilepsia 1986;27 Suppl 3:S1-20.

2. Alqahtani S, Alzaidi T, Alotaibi M, Alsultan A. Estimation of phenytoin pharmacokinetic parameters in Saudi epileptic patients. Pharmacology
2019;104:60-6.

3. Moon HJ, Jeon B. Can therapeutic-range chronic phenytoin administration cause cerebellar ataxia? J Epilepsy Res 2017;7:21-4.

4. Aring $C D$, Rosenbaum M. Ingestion of large doses of dilantin sodium. Arch Neur Psych 1941;45:265-70.

5. Utterback RA. Parenchymatous cerebellar degeneration complicating diphenylhydantoin (dilantin) therapy. Arch Neur Psych 1958;80:180-1.

6. Patocka J, Wu Q, Nepovimova E, Kuca K. Phenytoin - an anti-seizure drug: overview of its chemistry, pharmacology and toxicology. Food Chem Toxicol 2020;142:111393.

7. Craig S. Phenytoin poisoning. Neurocrit Care 2005;3:161-70.

8. Ney GC, Lantos G, Barr WB, Schaul N. Cerebellar atrophy in patients with long-term phenytoin exposure and epilepsy. Arch Neurol 1994;51: 767-71.

9. De Marcos FA, Ghizoni E, Kobayashi E, Li LM, Cendes F. Cerebellar volume and long-term use of phenytoin. Seizure 2003;12:312-5.

10. Shanmugarajah PD, Hoggard N, Aeschlimann DP, et al. Phenytoin-related ataxia in patients with epilepsy: clinical and radiological characteristics. Seizure 2018:56:26-30.

11. Seil FJ. The changeable nervous system: studies on neuroplasticity in cerebellar cultures. Neurosci Biobehav Rev 2014;45:212-32.

12. Lawrenson $C$, Bares $M$, Kamondi $A$, et al. The mystery of the cerebellum: clues from experimental and clinical observations. Cerebellum Ataxias 2018;5:8.

13. Mueller EW, Boucher BA. Fosphenytoin: current place in therapy. J Pediatr Pharmacol Ther 2004;9:265-73.

14. Govind E, Naik R, Srinivas BJ, Mehdi I. Case of fosphenytoin induced acute cerebellar dysfunction and pituitary bleed causing central diabetes insipidus in a case of AML M2 during allogenic hematopoietic stem cell transplant. Int I Pharmacol Clin Sci 2012;1:111-4.

15. McLachlan RS. Pseudoatrophy of the brain with valproic acid monotherapy. Can J Neurol Sci 1987;14:294-6.

16. Iorga A, Horowitz BZ. Phenytoin Toxicity [Internet]. Treasure Island: StatPearls Publishing, 2020 [cited 2020 Nov 13]. Available at : https://www.ncbi.nlm.nih.gov/books/NBK482444/.

17. Brostoff JM, Birns J, McCrea D. Phenytoin toxicity: an easily missed cause of cerebellar syndrome. J Clin Pharm Ther 2008;33:211-4.

18. Logan WJ, Freeman JM. Pseudodegenerative disease due to diphenylhydantoin intoxication. Arch Neurol 1969;21:631-7. 